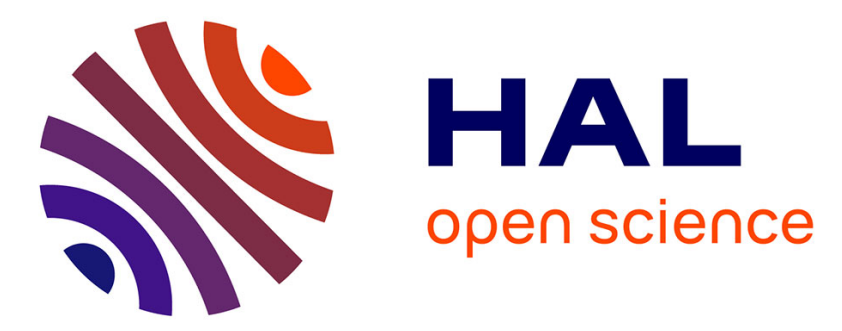

\title{
Biosensors in Diabetes : how to get the most out of evolution and transpose it into a signal
}

Sylvie Renaud, Bogdan Catargi, Jochen Lang

\section{To cite this version:}

Sylvie Renaud, Bogdan Catargi, Jochen Lang. Biosensors in Diabetes: how to get the most out of evolution and transpose it into a signal. IEEE pulse, 2014, pp.30-34. hal-00993190

\section{HAL Id: hal-00993190 \\ https://hal.science/hal-00993190}

Submitted on 19 May 2014

HAL is a multi-disciplinary open access archive for the deposit and dissemination of scientific research documents, whether they are published or not. The documents may come from teaching and research institutions in France or abroad, or from public or private research centers.
L'archive ouverte pluridisciplinaire HAL, est destinée au dépôt et à la diffusion de documents scientifiques de niveau recherche, publiés ou non, émanant des établissements d'enseignement et de recherche français ou étrangers, des laboratoires publics ou privés. 


\title{
Biosensors in diabetes - how to get the most out of evolution and transpose it into a signal
}

\author{
Sylvie Renaud ${ }^{1}$, Bogdan Catargi ${ }^{2}$ and Jochen Lang ${ }^{3}$ \\ ${ }^{1}$ CNRS UMR 5218, Institut Polytechnique de Bordeaux, Talence, France \\ ${ }^{2}$ Hôpital St André, Université de Bordeaux, CNRS UMR 5248, Pessac, France \\ ${ }^{3}$ CNRS UMR 5248, Université de Bordeaux, Pessac, France
}

The increase in diabetes is a major threat to health and to economic development in the 21st century as stated by the UN in the Millennium Development Goals. In 2013380 million people had diabetes and almost 600 million are expected by 2035. Diabetes is the major cause of non-traumatic amputation and of blindness in developed countries. It is also an expansive disease. In the US one in five dollars for health care is spent on diabetes. Net losses in national income from diabetes amount to hundreds of billions international dollars in China or India. Clearly novel therapies are required.

The burden for the patients, their carers and the national economies drives the search for novel treatments as new technologies may provide crucial human and economic benefits. One recent approach is continuous health monitoring, such as continuous glucose monitoring (CGM). A striking example is the recent proposal by Google: to implant a biosensor into contact lenses to determine tear glucose levels and transmit the data to a pump in the patient's body. The pump will inject the required level of insulin, the lacking hormone. This device circumvents multiple daily blood tests and injections in certain types of diabetes. Is the problem solved by contact lenses? Can all diabetic patients quote Humphrey Bogart's Rick "Here's looking at you, kid" and get their blood values straight? Well, life may be more complex.

Let's have a look what is diabetes to grasp the problem. We distinguish two major forms of the disease: type 1 , caused by destruction of insulin-producing beta-cells in the pancreatic islets, and type 2, caused by insufficient insulin release and reduced efficacy of the hormone. Type 2, the major form, is found in elder patients and a panel of life style interventions and drug therapies are available. In contrast, type 1 declares earlier in life and needs hormone replacement therapy by insulin injections. As insulin is extremely potent and can cause brain damage and death by hypoglycemia, glucose levels have to be controlled very precisely, requiring blood samples to be taken, a cumbersome burden for patients. Although fewer patients are concerned by type 1 diabetes, still some 80.000 children develop the disease each year and numbers are on the rise. This situation provides a strong impetus to develop continuous glucose monitoring via sensors that determine the amount of insulin to be injected. A number of different physicochemical methods have been explored for non-invasive approaches: surface-enhanced Raman, thermal or impedance spectroscopy, fluorescence, reverse iontophoresis, photo-acoustic or electromagnetic sensing. In general, these approaches are elegant but suffer from lack in specificity, problems in miniaturization or provoke major local reactions impeding their use.

Accepted medical technology for continuous glucose monitoring uses the protein glucose oxidase that generates an oxidation current upon reaction with glucose. Grafting the enzyme on an electrode allows to determine the current and hence glucose levels. First clinical devices were employed in hospitals in the 1970 s leading to the concept of the artificial pancreas: an implantable device measures interstitial blood glucose and drives an insulin pump that delivers the hormone via a subcutaneous needle. The first portable continuous glucose monitors (CGM) to display real-time values became available in 2006. Recent data suggest that these devices not only lift an awesome burden from the patients but also improve their long-term health, an important argument for reimbursement by health insurances. They still show a number of drawbacks such as considerable lag-time and are less accurate in hypoglycemia, a situation that may be life-threatening. Moreover, they work fine in between meals, but are not able to cope automatically with the rapid increase in glucose after a meal. As they tend to respond late and overdose, this may lead to a down in blood glucose at later time points. A number of smart algorithms have been developed to improve CGM and insulin therapy. However, current devices work essentially as an open loop because of the difficulty to find the adequate pattern to master the complexity of life.

Can the biosensor at the base of the artificial pancreas be improved? Current approaches rely on a small building block of cells, a protein. Can we respond better to complexity if we step up one level in the "bio" part and move to cells? Indeed, the relevant cells or the micro-organ in our body has been shaped to its current function during half a billion of years by evolution. Thus part of the work is already done by nature and we may perform the next step, the bio engineering, in somewhat less time. With this concept in mind we started out recently to 
develop a novel type of a hybrid bio-electronic sensor in a multidisciplinary approach between dialectologists, biologists and microelectronics [1].

How do pancreatic islet cells work (see Fig. 1)? The insulin-producing beta-cells in islets completely metabolize glucose, in a way they count each carbon of the molecule, and produce a novel molecule, ATP that is in precise correlation to the glucose levels outside the cell. ATP in turn will regulate a particular ion channel that depolarizes the cell, the $\mathrm{K}_{\mathrm{ATP}}$, and allows calcium influx through calcium channels. Calcium influx will trigger the release of the precise amount of insulin, adapted to glucose levels. However, food and nutrient homeostasis is much more than sugar. The beta-cells will not only respond to and metabolize glucose, but also lipids and some amino acids and count their carbons. So different from current sensors, our $\beta$-cells release insulin whether we pinch a bit of our kid's cotton candy or have a foie gras. More importantly, the cell integrates also a number of other signals, such as different hormones, that reflect the state and activity of the body. Jogging or trying to catch the train? You need your glucose and should not secrete much insulin. To this end the adrenal glands release epinephrine, that inhibits ion channels thus reducing ion fluxes. Therefore the electrical behavior of the cells, their field potentials, is the first signal that integrates clues from different nutrients and takes the state of the whole body into account. Interestingly the islets have two different major cell populations: beta-cells that secrete insulin and bring glucose down, and $\alpha$-cells, that secrete glucagon, a hormone that liberates glucose from the liver. $\alpha$-cells behave exactly inverse of $\beta$-cells in electrical terms and recording both will considerably increase precision. If we manage to keep these cells alive for days to weeks and capture these very tiny electrical potentials, we may obtain a highly suitable signal to determine the demand for insulin.

We were able to record these signals in islets obtained from mice or most recently from human donors and found two distinct types of signals: short-lived action potentials, just as in nerve cells, lasting some tens of milliseconds, and slower undulations that we termed "slow waves". Whereas the action potentials represent the activity of a single cell, the slow waves represent the further effect of the cell-cell interactions as found in the islet micro-organ. The signals are finely tuned by glucose within the physiological range and fully regulated by the physiological levels of hormones [2]. Thus, cells have followed long-ago the trend that generally comes up in the development of sensors: the implementation of multi-parametric sensors to adapt to diverse lifestyle and the multitude of daily events, and to improve accuracy and safety, a major concern for the use in humans.

Recording quality is a challenge: it must be high enough to discriminate slight differences resulting from slow changes in the physiology. We used multi-electrode arrays (MEA) that contain multiple plates/electrodes to connect the cells to electrical circuitry (Fig. 2A-B) [3]. MEAs have typically an arrangement of 60 electrodes with a diameter between 10 and $20 \mu \mathrm{m}$. Cell preparations are cultured directly on the MEA. We designed custom integrated circuits able to process the 60 signals online and in parallel in analog then in digital mode. The circuits were embedded in an electronic board (Fig. 2C) providing on board data storage on SD cards and interfacing with standard peripherals using UART and VGA protocols. Data processing is of 2 types: first, conditioning of raw recorded data to extract significant signal markers (action potential and slow waves); second, evaluation of these markers to indicate changes in islets physiology. Thanks to the use of configurable circuits, evaluation can be adapted to the context and provides the user with quantitative information or go/no-go flags. Electronics were designed in low power submicron technology, sized to ensure continuous and real-time processing on up to 60 channels. A computer-interface is available to configure the system, launch recordings and store raw or processed data in user-specific formats.

Currently we are developing the technology for two distinct "lab-on-chip" applications: Diaßchip and Diaßsensor. Diaßchip will perform multi-parametric testing of islets, to be used outside the human body for a number of defined applications. For example, one treatment of unstable forms of type diabetes is transplantation of islets. Currently there is no quality control of donor islets that have to be transplanted within $6 \mathrm{~h}$. We have started a project with clinicians to compare transplantation outcome and islet quality. Another application concerns the differentiation of pluripotent stem cells into $\beta$-cells. If this differentiation becomes possible and the whole process can be mastered reliably, diabetic patients, either of type 1 or of type 2, can be definitively cured by transplantation of these cells. Current procedures require the treatment of stem cells with very complex mixtures of biological factors, and their composition often changes daily. Diaßchip will be able to monitor their development on a non-invasive natural base and may also provide a useful element in quality control.

Our long-term goal, Diaßsensor, is even more ambitious and of high risk: the development of a novel and physiological sensor for an artificial pancreas and to free type 1 diabetic patients from cumbersome programming and complications inherent to glucose sensing only. Whereas islet transplantation needs several donor million islets, which are difficult to obtain, the sensor requires only a few dozen. Diaßsensor is essentially the core of a 
closed-loop controlling insulin delivery depending on the patient's state and nutrient levels. Closed-loop control is very common in machinery: cruise control in cars, temperature control at home (fig. 3A). Artificial pancreas based solely on CGM is a step towards closed-loop control for diabetes but still misses essential points: glucose-only dependence, open-loop via the CGM (fig. 3B). We expect Diaßsensor to act as a real closed-loop with few, ideally none - user inputs. It will therefore necessitate an extremely robust feedback control, able to handle both fast and slow changes in the patient's physiology. The whole loop signal processing (conditioning, evaluation and feedback control) has to be finely designed and to be flexible enough to suit most patients (Fig. $3 \mathrm{C}$ ). Diaßsensor will require highly performing microelectronic technology, as the implanted device should be of minimum size and weight and ultra-low power. Diaßsensor implies the use of cells that are foreign to the patient's body with the risk of immune reactions. Recently devices have been engineered that retrieve plasma from blood vessels and are positioned outside the patient's body. Considerable progress has also been achieved in material sciences to provide adequate support that ensures long survival of the cells. This field is a very active area and considerable further progress can be expected in the near future.

It took almost fifty years to develop glucose sensors adapted to therapy and they have revolutionized diabetes care. It is now time to go beyond the use of few constituents of cells, such as proteins, already marvels of evolution, and to exploit nature's more complex developments as given by a micro-organ such as the islets. Expertise gained in this field may also help neuroscience to come up with operational biosensors and artificial organ parts to provide relieve to a number of inborn or traumatic pathologies. In any case, the diabetes field, with its longstanding and well organized scientific, medical and patient communities, should be able to benefit from interdisciplinary work in biomedical engineering.

\section{References:}

1. Bornat, Y., M. Raoux, Y. Boutaib, F. Morin, G. Charpentier, J. Lang, and S. Renaud. Detection of Electrical Activity of Pancreatic Beta-cells Using Micro-electrode Arrays. in Electronic Design, Test and Application, 2010. DELTA '10. Fifth IEEE International Symposium on. 2010.

2. Nguyen, Q.V., A. Caro, M. Raoux, A. Quotb, J.-B. Floderer, Y. Bornat, S. Renaud, and J. Lang. A novel bioelectronic glucose sensor to process distinct electrical activities of pancreatic beta-cells. in Engineering in Medicine and Biology Society (EMBC), 2013 35th Annual International Conference of the IEEE. 2013.

3. Raoux, M., Y. Bornat, A. Quotb, B. Catargi, S. Renaud, and J. Lang, Non-invasive long-term and real-time analysis of endocrine cells on micro-electrode arrays. J Physiol, 2012. 590: p. 1085-91. 


\section{Figure Legends}

Figure 1. Sensing the demand in insulin: Biological sensors versus electrochemical sensor. Left part indicates the sensing of the demand in insulin by pancreatic beta-cells in response to nutrients and hormones, whereas current glucose-sensing electrodes respond only to glucose. Details see text.

Figure 2 Two young members of the lab looking at a MEA. Note screen display of recorded activities.

Figure 3 Open and closed loops. A. In engineering, in a closed-loop control system, a sensor monitors the physical system output and feeds the data to a controller which adjusts the control as necessary to maintain the desired system output, i.e. the "reference ". A closed-loop system is generally modelled by the mathematical description of its dynamics. In blue, the example of the closed-loop temperature regulation when using a steam air heater? B. The artificial pancreas is a system that measures blood glucose levels using a continuous glucose monitoring (CGM) device, and transmits this information (wired or RF) to an controlled insulin pump (in grey) that calculates and releases the required amount of insulin into the body. C. Diaßsensor is a dynamic closed-loop system able to regulate insulin release based on the physiological response of healthy islets to nutrients and hormones from a diabetic patient. All loop items (biosensor, controller, insulin pump grey area) are to be integrated in low power implanted devices. External control via a PC or smartphone application will ensure inocuity and will control calibration phases.

Figure 4: Current prototype. Photographs of $(A)$ the center of a MEA with an array of 60 TiN electrodes $(10 \mu \mathrm{m} \times 10 \mu \mathrm{mm}, 100 \mu \mathrm{m}$ spacing); wires connect the electrodes to external pads for electronic connection; cultured islets cells are visible on the array. (B) a MEA device (same as in (A). The circular reservoir for the culture is stacked at the center of expoxy laminated sheet. A ring of 60 pads allows connections to external circuits.(C) The electronics board for configurable recording an real time processing of islet signals. It can be used as a stand-alone device without any peripherals. Pre- and post-processed data are stored on SD cards on board. Configuration is possible using the on-board buttons and switches or using a dedicated software on an eternal PC. Serial and parallel outputs are avaiable for signals display or post-processing. 


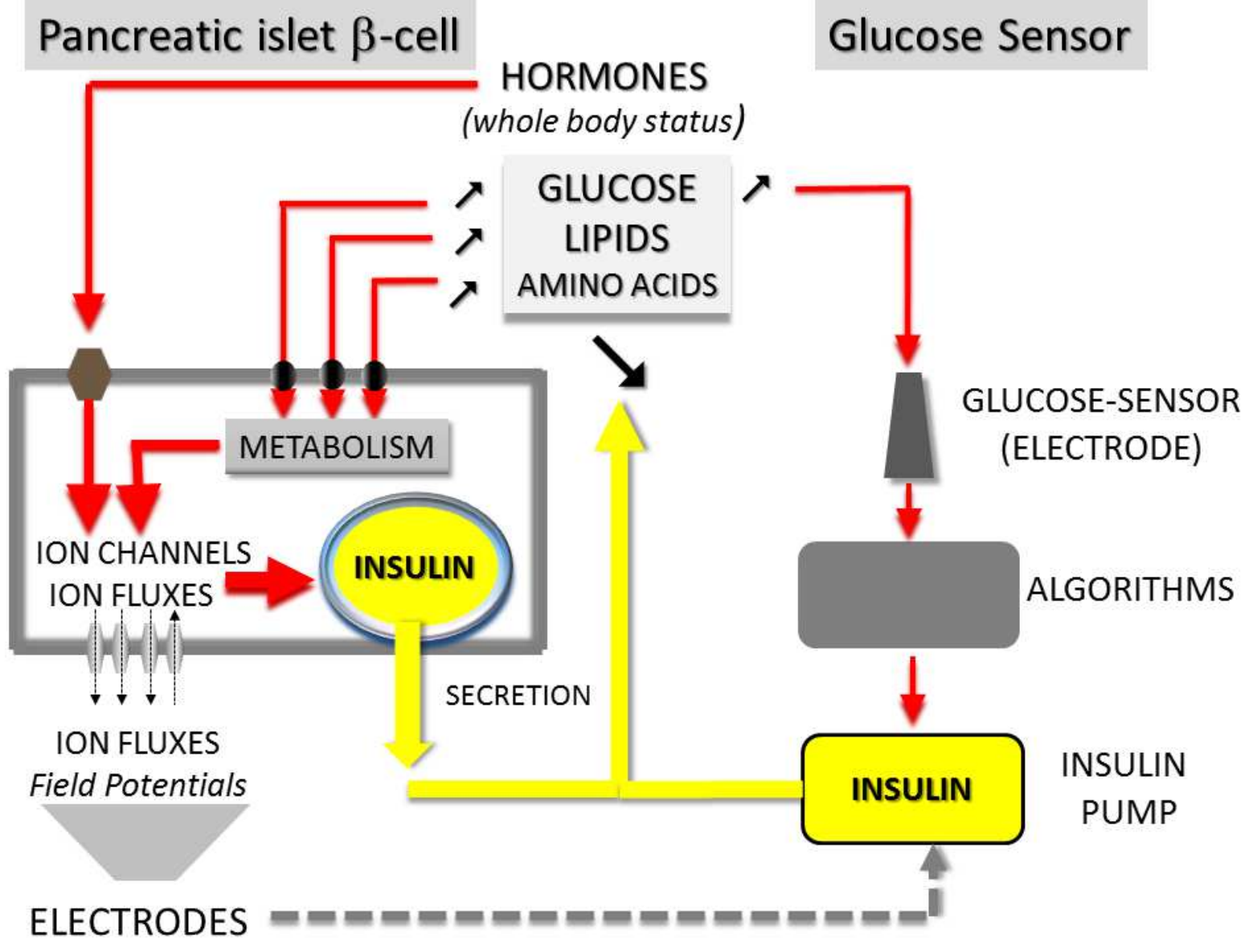




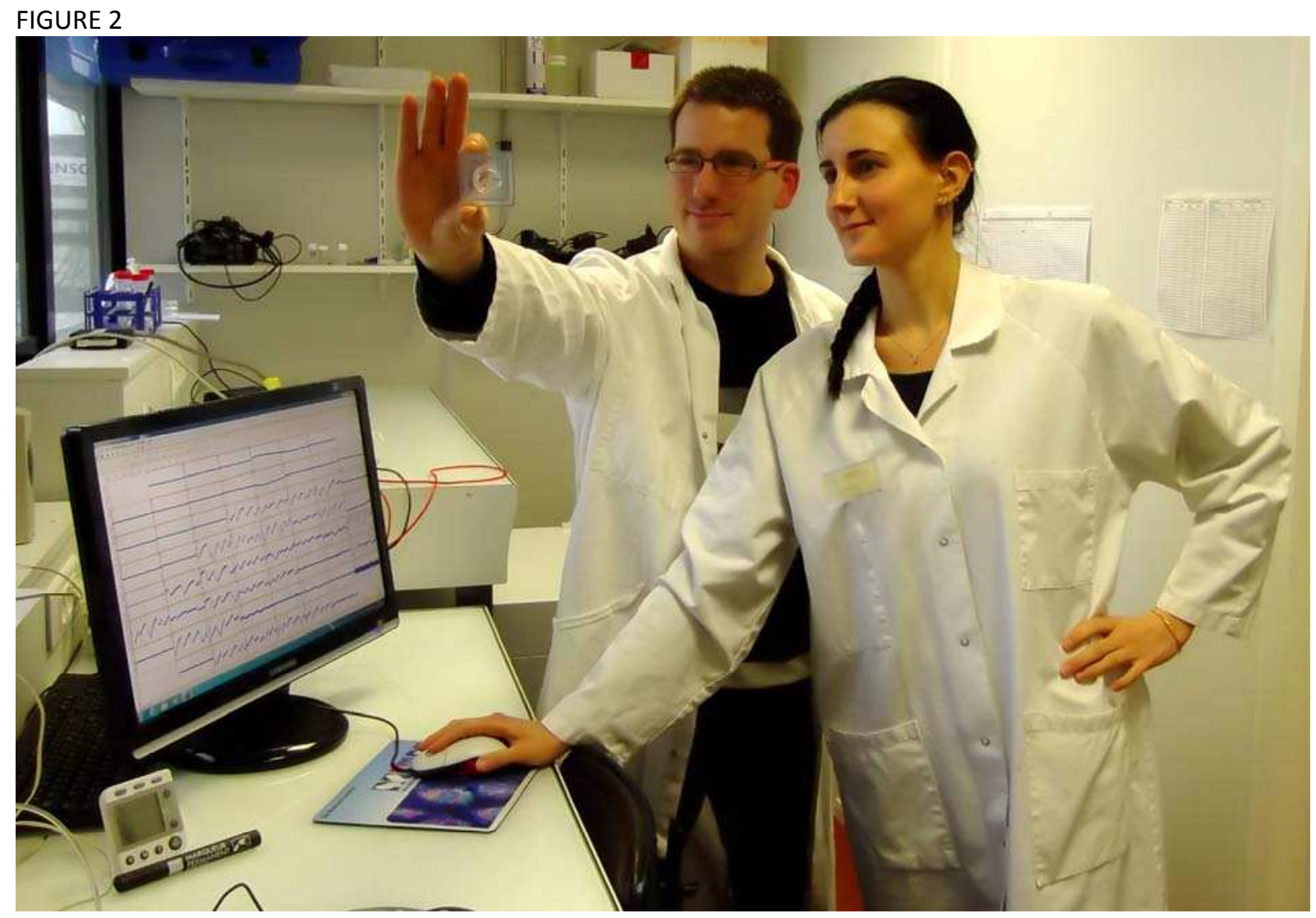


A

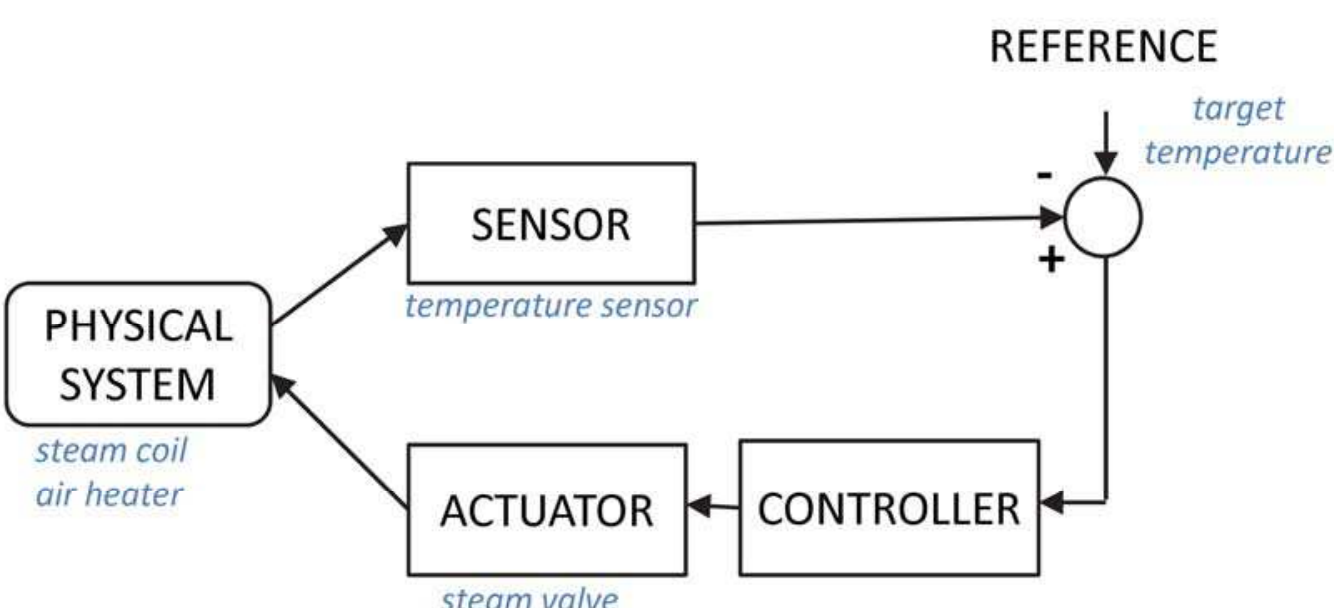

B
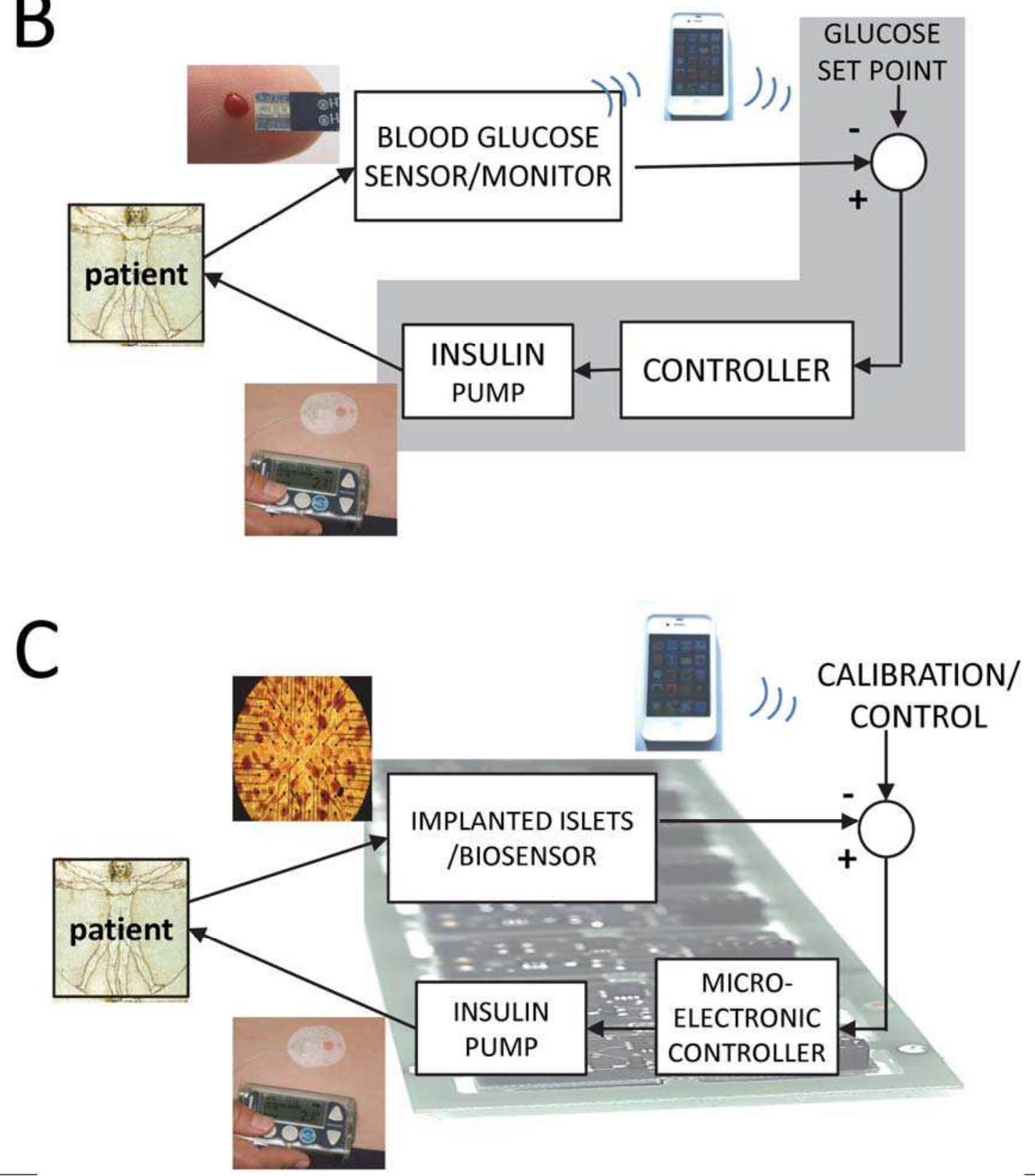
FIGURE 4
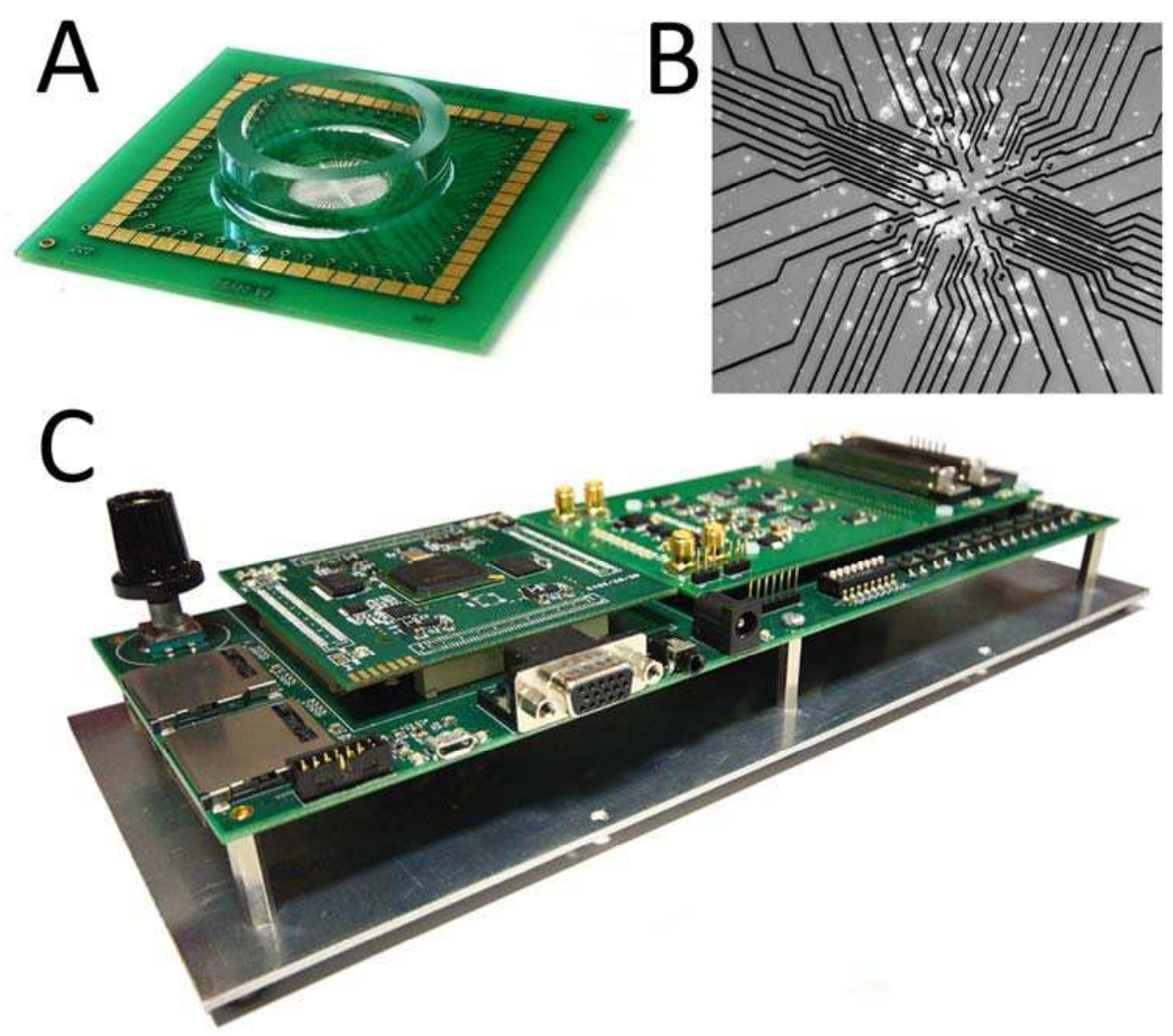